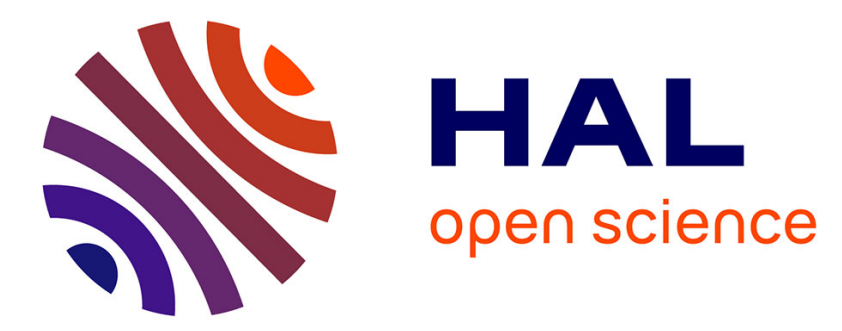

\title{
Response of a two-dimensional liquid foam to air injection: Influence of surfactants, critical velocities and branched fracture
}

\author{
Imen Ben Salem, Isabelle Cantat, Benjamin Dollet
}

\section{To cite this version:}

Imen Ben Salem, Isabelle Cantat, Benjamin Dollet. Response of a two-dimensional liquid foam to air injection: Influence of surfactants, critical velocities and branched fracture. Colloids and Surfaces A: Physicochemical and Engineering Aspects, 2013, 438, pp.41-46. 10.1016/j.colsurfa.2013.01.013 . hal-00905596

\section{HAL Id: hal-00905596 \\ https://hal.science/hal-00905596}

Submitted on 29 Jan 2014

HAL is a multi-disciplinary open access archive for the deposit and dissemination of scientific research documents, whether they are published or not. The documents may come from teaching and research institutions in France or abroad, or from public or private research centers.
L'archive ouverte pluridisciplinaire HAL, est destinée au dépôt et à la diffusion de documents scientifiques de niveau recherche, publiés ou non, émanant des établissements d'enseignement et de recherche français ou étrangers, des laboratoires publics ou privés. 


\title{
Response of a two-dimensional liquid foam to air injection: influence of surfactants, critical velocities and branched fracture
}

\author{
Imen Ben Salem, Isabelle Cantat, Benjamin Dollet \\ Institut de Physique de Rennes, UMR 6251 CNRS/Université de Rennes 1, Campus \\ Beaulieu, Bâtiment 11A, 35042 Rennes Cedex, France
}

\begin{abstract}
Experiments where air is injected into a foam confined in a Hele-Shaw cell are convenient to study the rheology of foams far from the quasistatic regime, and their limit of stability. At low overpressure, the injected air forms a ductile crack, whereas at high overpressure, it breaks the foam like a brittle material. We present new results in this configuration, complementary with previous studies. We show that air injection is slowed down for surfactants giving incompressible interfaces instead of mobile ones. The injection rate is quantitatively captured by a simple model balancing the air overpressure with known foam/wall friction laws for incompressible interfaces. We also revisit the critical velocity criteria for the injected air proposed by Arif et al. [1]. The upper bound of velocity in the ductile regime, based on the resistance of soap films against wall friction, is shown to hold much better for mobile than for incompressible interfaces. The propagation speed of shear waves is confirmed to be a good lower bound for the velocity in the brittle regime, provided the motion of all liquid within the foam is accounted for. Finally, a short description of branching in the fragile regime is given.
\end{abstract}

Keywords: liquid foam, air injection, foam/wall friction, ductile, fragile, branching

Email address: benjamin.dollet@univ-rennes1.fr, Phone: +33223235038 (Benjamin Dollet) 


\section{Introduction}

Liquid foams are a typical example of complex fluids: they can exhibit elastic, plastic or viscous response, depending on the external forcing [2]. Understanding this intricate macroscopic behaviour in relation with considerations at the scale of single bubbles and films motivates active research $[3,4,5]$, with open questions on e.g. shear localisation [6] or nonlocal effects [7]. Still, even if viscous effects are significant at the macroscopic scale, most of these studies remain in a quasistatic regime for the local structure; that is, deviations from the equilibrium rules for the film network (the so-called Plateau rules) remain negligible. However, it is a question of high applied and fundamental relevance to understand the rheology of foams as they depart from quasistatics, up to the conditions for which they fail. For instance, this is important to quantify energy absorption associated to blast wave mitigation by aqueous foams $[8,9,10]$, and to study flows of soap films at high velocity $[11,12]$.

A good setup to study rheology of foam far from quasistatics consists of injecting air into a foam confined in a Hele-Shaw cell. Initially motivated by pattern formation $[13,14]$, this configuration was shown by Hilgenfeldt and coworkers to be ideal to study the limit of stability of a flowing foam $[1,15]$. They showed that the injected air can propagate either in a ductile regime, pushing bubbles apart by plastic rearrangements without bursting; or in a fragile regime, breaking series of soap films to form narrow cracks, like fracture in brittle materials [16]. Most interestingly, they showed that the propagation velocity of the advancing front of injected air has an upper bound in the ductile regime, and a lower bound in the fragile one, with a large gap of "forbidden" velocities in between. Recently, we performed a detailed study of the ductile regime [17]. We quantified the swelling rate of the air pocket injected in the foam, as a function of its overpressure, with a comprehensive explanation of the role of all control parameters: cell gap, bubble size, foam area (i.e. the total area covered by foam), in the frame of a model balancing the overpressure with foam/wall friction. We have shown that the air pocket displays a fingering instability, which we were able to quantitatively explain by a linear stability analysis of the aforementionned model.

In this paper, we wish to give complementary results on the response of a confined foam to air injection. First, we will study the influence of surface rheology, yet unexplored in the configuration of air injection in foam. Two 
limiting regimes have been identified [3]: the "mobile" limit, where surfactants confer to the gas/liquid interfaces a negligible viscoelasticity, and the incompressible (or "immobile") limit, where surfactants at interfaces display a high viscoelasticity, hence a strong resistance to interfacial compression. In terms of hydrodynamic boundary conditions, the "mobile" limit gives free shear, and the incompressible one no slip. In [17], we used a sodium dodecyl sulfate (SDS) solution, which is in the "mobile" limit. To study the opposite interfacial behaviour, we will show results with a surfactant mixture giving incompressible interfaces. Second, we will test the critical velocities proposed by Hilgenfeldt and colleagues $[1,15]$, namely the maximal velocity of ductile fracture and the minimal velocity of brittle fracture. Third, we will describe branching effects in the regime of fragile fracture.

\section{Materials and methods}

The principle of the experiments is explained in full detail in [17]. Briefly, the flow cell is made of two horizontal circular glass plates of diameter $30 \mathrm{~cm}$ separated by three spacers of thickness $e=0.6 \mathrm{~mm}$. (see Tab. 1 for a table of the notations introduced throughout the paper). The top plate is simply set on the spacers and the lateral boundary of the cell is open. Beside the flow cell, a flat open container is entirely filled with a soap solution. The foam is prepared by blowing air at a controlled flux controlled by a syringe pump through a needle in the container. Foam accumulates at the free surface of the liquid. We take the top plate, and place it just above the container, in contact with the foam. Most of the foam is then transferred to the plate after withdrawal. The top plate is then set on the spacers, and the foam is squeezed between the two plates. The bubble size and the spacer height are chosen in order to obtain a single monolayer of bubbles. The foaming solution reservoir is weighed before and after the foam collection by the top plate; the difference is a measure of the mass $m$ of liquid contained within the foam.

In this paper, we use either a fresh foaming solution of SDS at concentration $10 \mathrm{~g} / \mathrm{L}$ and glycerol $10 \%$ wt in ultra pure water, or a mixture of sodium lauryl-dioxyethylene sulfate (SLES), cocoamidopropylbetaine (CAPB) and myristic acid (MAc), following the protocol proposed in [18]. We have measured the viscosity of the solutions $\eta$, the surface tension $\sigma$, and the surface modulus $E_{D}$ quantifying the interfacial dilatational viscoelasticity [3]. For the SDS solution, $\eta=1.2 \mathrm{mPa} . \mathrm{s}, \sigma=36.8 \pm 0.3 \mathrm{mN} / \mathrm{m}$, and $E_{D}$ is 


\begin{tabular}{|l|l|}
\hline$a$ & radius of curvature of the Plateau borders \\
$A$ & area of the injected bubble \\
$A_{t}$ & opening speed of the injected bubble \\
$A_{\text {tot }}$ & initial area covered by the foam \\
$b$ & side of a hexagon \\
$c_{L}$ & propagation velocity of longitudinal waves (speed of sound) \\
$c_{S}$ & propagation velocity of shear waves \\
$C_{\mathrm{I}, \text { film }}$ & dimensionless constant in $(3)$ \\
$C_{\mathrm{I}, l i n e}$ & dimensionless constant in $(3)$ \\
Ca & capillary number \\
$e$ & cell gap \\
$E_{D}$ & dilatational surface modulus [3] \\
$f_{3}$ & dimensionless parameter in (3) \\
$f_{v}$ & friction force per unit length of a Plateau border \\
$\vec{F}_{v}$ & friction force per unit area of the foam \\
$L$ & equivalent radius of the bubbles \\
$L^{\prime}$ & typical length between two rows of bubbles \\
$m$ & mass of liquid contained in the foam \\
$n$ & exponent of the velocity in the friction force \\
$N$ & number of bubbles within the foam \\
$P_{0}$ & overpressure of the injected gas \\
$\bar{S}$ & mean bubble area \\
$t$ & time \\
$\vec{v}$ & velocity field \\
$v_{\text {max }}$ & maximal velocity of ductile propagation \\
$v_{\min }$ & minimal velocity of fragile propagation \\
$\delta$ & boundary layer thickness \\
$\delta S / S$ & relative area variation (for the measurement of $\left.E_{D}\right)$ \\
$\delta \sigma / \sigma$ & relative variation of surface tension \\
$\eta$ & viscosity of the solution \\
$\kappa$ & curvature of the lamella \\
$\phi_{\ell}^{\prime}$ & prefactor in the friction force (1) \\
$\rho$ & liquid density \\
\hline & surface tension \\
\hline & bubble/wall stress \\
\hline & pressure gradient \\
\hline
\end{tabular}

Table 1: Table of the notations used in this paper. 
below $1 \mathrm{mN} / \mathrm{m}$. It is thus in the so-called "mobile" limit [3]. For the SLES/CAPB/MAc mixture, $\eta=1.0 \mathrm{mPa} . \mathrm{s}, \sigma=22.4 \pm 0.3 \mathrm{mN} / \mathrm{m}$, and $E_{D}=216 \mathrm{mN} / \mathrm{m}$ for a frequency of $0.2 \mathrm{~Hz}$ and a relative area variation $\delta S / S_{0}=1 \%$. It is thus in the so-called incompressible limit [3].

The foam structure is lit with a circular neon tube put between the bottom plate and a black plate, and is recorded with a high-speed camera. The bubble edges appear in white on a black background. In this paper, images are inverted for a better rendering. The foam, i.e. the assembly of all the bubbles except the large bubble formed by the injected air in the ductile regime, that we call the injected bubble, is made of $N$ bubbles of mean area $\bar{S}$. We measure the initial area $A_{\text {tot }}$ occupied by the foam, before air injection. If there is no film rupture, this parameter is constant with time, as are $N$ and $\bar{S}$. We take as an effective bubble size the equivalent radius $L=\sqrt{\bar{S}} / \pi$. The area of the injected bubble is denoted by $A(t)$ and is directly extracted from the image processing. The liquid fraction is obtained as $\phi_{\ell}=m / \rho e A_{\text {tot }}$, with $\rho=1 \mathrm{~g} / \mathrm{cm}^{3}$ the liquid density. In most of our experiments, it is close to $2 \%$. We also refer to the radius of curvature $a$ of the Plateau borders, which can be deduced from the other parameters as (see [17] for details): $a=\sqrt{m /\{\rho N[\pi(4-\pi) L+(2 \sqrt{3}-\pi) e]\}}$.

The bottom plate is drilled in the middle by a hole to allow gas injection. We perform our experiments at controlled pressure; for this, we use a large buffer tank of gas that we connect to the cell to blow gas at the centre of the foam. An electrovalve allows for a rapid opening of this gas reservoir, within $5 \mathrm{~ms}$. The overpressure in the flowing gas $P_{0}$ is measured by a differential pressure transducer, with an accuracy of $5 \mathrm{~Pa}$. As the typical pressure is of the order of a few thousands of Pa or less, the gas can be considered as incompressible.

\section{Influence of surfactants on the swelling rate}

To illustrate the influence of surface rheology, we compare two experiments, one with the SDS solution and one with the SLES/CAPB/MAc mixture, with the same foam mass, bubble size and pressure (within 10\%); only the foam area differs significantly between the two experiments. Fig. 1 shows that the injected bubble grows much faster in the case of the SDS solution than for the SLES/CAPB/MAc mixture. This is not surprising: bubble/wall friction has been shown to be much higher in the case of incompressible in- 


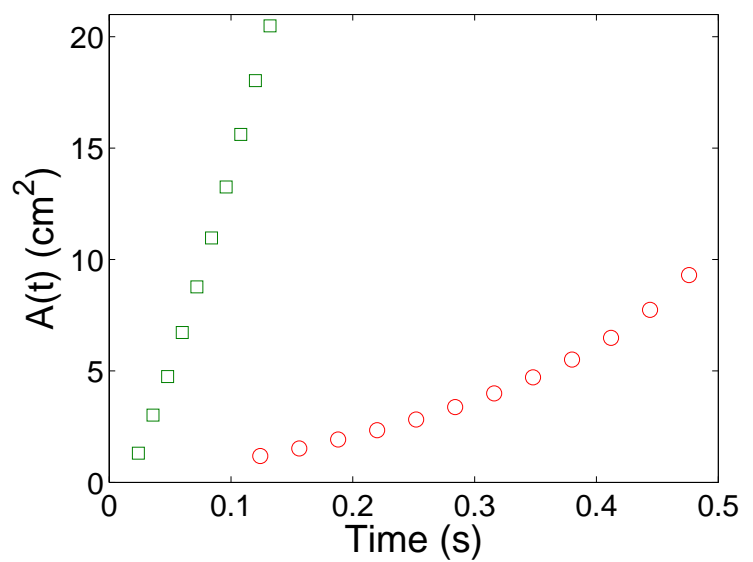

Figure 1: Time evolution of the central bubble area $A(t) . \square$ : SDS solution (experimental parameters: $\left.m=0.14 \mathrm{~g}, L=0.22 \mathrm{~cm}, P_{0}=460 \mathrm{~Pa}, A_{\text {tot }}=44.1 \mathrm{~cm}^{2}\right)$, $\circ$ : SLES/CAPB/MAc mixture (experimental parameters: $m=0.13 \mathrm{~g}, L=0.20 \mathrm{~cm}$, $\left.P_{0}=483 \mathrm{~Pa}, A_{\text {tot }}=76.2 \mathrm{~cm}^{2}\right)$.

terfaces, hence at given overpressure, the dynamics is expected to be slower for such interfaces.

The data can be compared to the model that we developped in [17], where we assumed a radially symmetric opening for simplicity. The overpressure is the driving force for opening, and dissipation only comes from bubble/wall friction. Then, defining a factor $\xi^{\prime}$ such that:

$$
\vec{F}_{v}=-\xi^{\prime} v^{n-1} \vec{v}
$$

is the friction force per unit area of the foam ( $\vec{v}$ is the 2D velocity field, and $n$ a characteristic exponent), the opening speed was shown to be constant under the assumption $A \ll A_{\text {tot }}$ :

$$
A_{t}=\lim _{A / A_{\mathrm{tot}} \rightarrow 0}=2 \pi^{(1+n) / 2 n}\left[\frac{(1-n) P_{0}}{\xi A_{\mathrm{tot}}^{(1-n) / 2}}\right]^{1 / n} .
$$

For a given experiment, $A_{t}$ is determined as the slope of the best affine fit of the $A(t)$ curve; possible nonlinearities, like that observed in Fig. 1, where the opening speed increases with time, are included as error bars. The relative uncertainty on $A_{t}$ implied by this procedure remains below $15 \%$. For experiments for which $L, \phi_{\ell}$ and $A_{\text {tot }}$ are kept as constant as possible, Fig. 2 shows that the overpressure is an increasing function of the opening speed. 


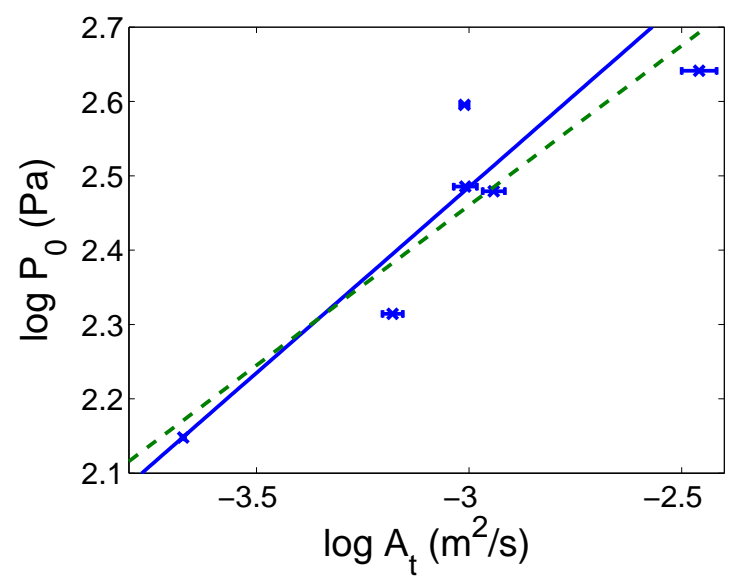

Figure 2: Plot of $\log P_{0}$ (with $P_{0}$ in $\mathrm{Pa}$ ) as a function of $\log A_{t}$ (with $A_{t}$ in $\mathrm{cm}^{2} / \mathrm{s}$ ), for the SLES/CAPB/MAc mixture. The other parameters are: $L=1.80 \pm 0.12 \mathrm{~mm}$, $\phi_{\ell}=0.023 \pm 0.004$ and $A_{\text {tot }}=102 \pm 19 \mathrm{~cm}^{2}$, where the standard deviations comes from the dispersion between the different experiments. The plain line is prediction (2) with a factor $\xi^{\prime}$ given by (4). The dashed line if the best affine fit of the data; its slope is $0.43 \pm 0.10$.

To compare experiments and theory, we determine $\xi^{\prime}$ and $n$ in (2) using the measurements of Denkov et al. [19], who have proposed a frictional stress for incompressible interfaces:

$$
\tau_{w}=\frac{\sigma}{L}\left(1.25 C_{\mathrm{I}, \mathrm{film}} \mathrm{Ca}^{1 / 2} \frac{f_{3}^{1 / 4}}{\sqrt{1-f_{3}^{1 / 2}}}+2.1 C_{\mathrm{I}, \text { line }} \mathrm{Ca}^{2 / 3}\right) f_{3}^{1 / 2},
$$

where $C_{\mathrm{I}, \mathrm{film}}=3.7$ and $C_{\mathrm{I}, \mathrm{line}}=3.5$ are two empirical constants, and $f_{3}=$ $1-3.2\left[\left(1-\phi_{\ell}\right) / \phi_{\ell}+7.7\right]^{-1 / 2}$, which is the relative area of the walls covered by the wetting films, takes into account the effect of the liquid fraction. The parameter $\mathrm{Ca}=\eta v / \sigma$ is the capillary number. This frictional stress is simply related to the friction force per unit area of the foam by: $\vec{F}_{v}=$ $-2 \tau_{w} \vec{v} / v$, where the factor two accounts for the presence of the top and bottom confining plates.

The two terms in the right-hand side of (3) are respectively the contributions from the wetting films, and from the Plateau borders. In our experiments, $v$ remains below $20 \mathrm{~cm} / \mathrm{s}$ (see Sec. 4 ), hence Ca is below $10^{-2}$, and $\phi_{\ell}$ is close to $2 \%$, for which $f_{3}^{1 / 4} / \sqrt{1-f_{3}^{1 / 2}}=1.8$. Therefore, the dom- 

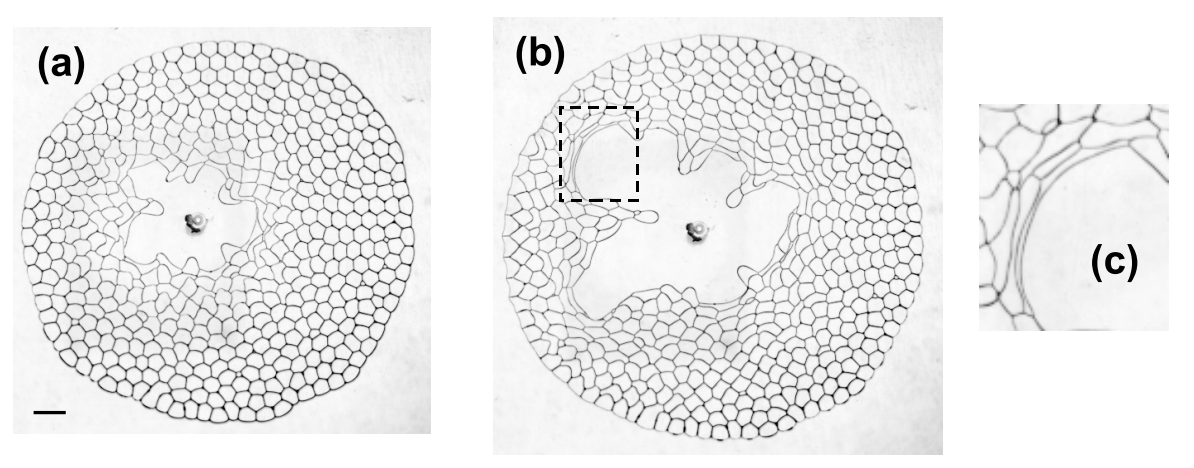

Figure 3: (a) Snapshot of a foam made of SLES/CAPB/MAc mixture subjected to air injection through the central nozzle. The bar scale is $1 \mathrm{~cm}$ in reality. (b) Same foam $164 \mathrm{~ms}$ later. (c) Zoom of the rectangular box drawn in (b) with a dashed frame.

inating contribution is that of the wetting films. Hence, comparing (1) and (3), we get $n=1 / 2$, and:

$$
\xi^{\prime}=\frac{\sigma}{L}\left(\frac{\eta}{\sigma}\right)^{1 / 2}\left(2.5 C_{\mathrm{I}, \mathrm{film}} \frac{f_{3}^{1 / 4}}{\sqrt{1-f_{3}^{1 / 2}}}+4.2 C_{\mathrm{I}, \mathrm{line}} \mathrm{Ca}^{1 / 6}\right) f_{3}^{1 / 2} .
$$

We plug this expression in (2), and we plot the corresponding prediction of $P_{0}$ versus $A_{t}$ together with the experimental data in Fig. 2 . We find a quantitative agreement. This is somewhat surprising, given the crude assumption of axisymmetry of the model: actually, as already observed for the SDS solution [17], the injected bubble displays a fingering instability (Fig. 3a,b). The good agreement gives a further confirmation of the incompressible friction force proposed in [19]. Furthermore, fitting our data in Fig. 2 by a power law gives an exponent $0.43 \pm 0.10$, which is compatible with 0.5 , but not with $2 / 3$, within error bars. The exponent 0.5 is typical of incompressible interfaces, although not in the full range of parameters [20], whereas the exponent $2 / 3$ corresponds to "mobile" interfaces. The exponent is therefore another manifestation of the difference between the two kind of interfacial behaviours.

\section{Critical velocities}

We now consider the critical velocities introduced by Hilgenfeldt and colleagues [1]. They have proposed that the speed of propagation of the ductile 
fracture, $i$. e. the fastest speed of the injected bubble in our case, cannot exceed a certain threshold, for which friction forces destabilise the Plateau borders in contact with the wall. Based on observations, they have also proposed that the propagation of fragile cracks cannot be less than the speed of sound $c$ in a 2D foam. Before proceeding, let us clarify the latter assertion: they actually refer to the velocity of shear waves $c_{S}$, and not that of longitudinal waves $c_{L}$, which would be the most usual meaning of the "speed of sound" (and the only meaning in fluid media). Given the huge difference between the shear modulus (or order $\sigma / L$ ) and the compression modulus (of order the atmospheric pressure) in a foam, we have $c_{S} \gg c_{L}$. We now test these criteria in the case of the two surfactant solutions.

\subsection{Maximal velocity in the ductile regime}

For foams made of a SDS solution, we have shown in [17] that the velocity of ductile propagation does not exceed a critical velocity $v_{\max }$, which is given in good approximation by the criterion:

$$
2 \sigma=f_{v},
$$

where $f_{v}$ is the friction force per unit length of a Plateau border sliding along a wall, given by Eq. (4.17) in [17]. We reproduce the experimental data, already shown in Fig. 7 in [17], in Fig. 4a for convenience. We recall the interpretation of this criterion: at low velocity, the friction of the Plateau border is compensated by the traction of the lamella (i.e. the film across the plates) attached to it. This traction force equals $2 \sigma$, the factor two coming from the presence of the two gas/liquid interfaces bounding the lamella. If $f_{v}$ exceeds $2 \sigma$, the traction cannot compensate the wall friction, and the lamella bursts $[21,11]$.

For the SLES/CAPB/MAc mixture, we have found that there exists a maximal velocity that the foam can sustain without breakage, as for the SDS solution. The measured maximal velocities are plotted as a function of the liquid fraction in Fig. 4b; they are typically five times lower than for SDS.

To try to explain the obtained values, we cannot extend directly the criterion (5). This was valid for "mobile" interfaces, where bubble/wall friction is localised at the Plateau borders (more precisely, in the transition regions between the Plateau borders and the flat wetting films along the walls), hence the notion of a friction force per unit length of Plateau border is meaningful. 

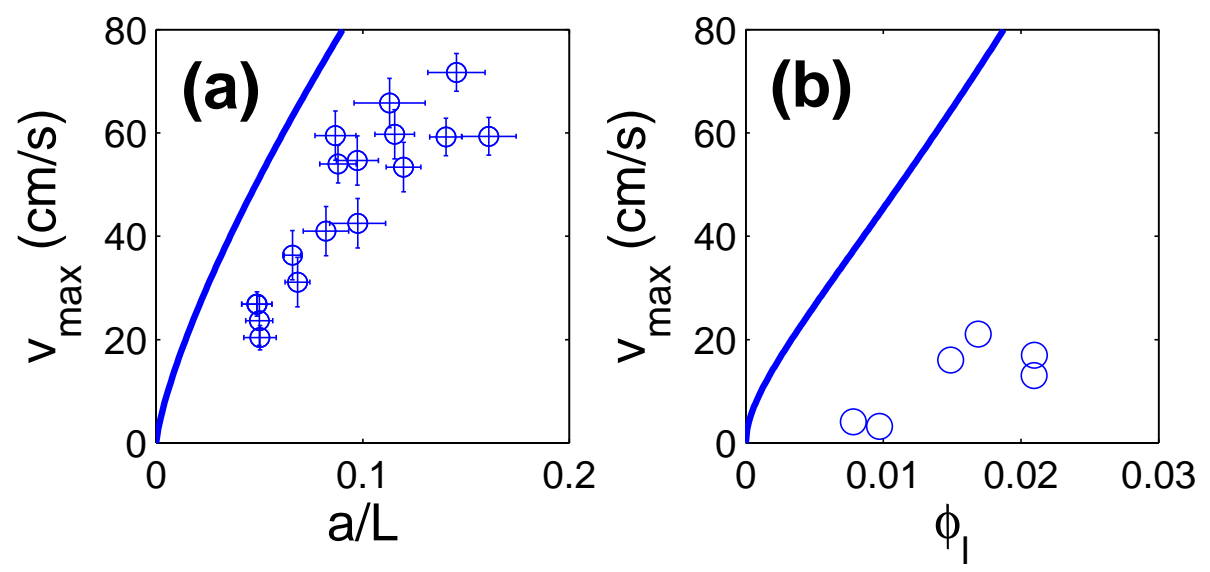

Figure 4: Experimental maximal velocity in the ductile regime, (a) as a function of $a / L$ for the SDS solution, and (b) as a function of $\phi_{\ell}$ for the SLES/CAPB/MAc mixture. The curve in (a) is the prediction (5), and the curve in (b) is the prediction (6); see text for details.

This is not the case for incompressible interfaces, where most of the friction occurs within the wetting films. However, we can modify the criterion as follows: the pressure difference across a lamella cannot exceed $2 \sigma \kappa$, with $\kappa$ the maximal possible curvature of the lamella. Since the lamella must remain attached to a top and a bottom Plateau border, the most curved shape is a half-circle tangent to the top and bottom plates, hence $\kappa=2 / e$. Hence, the pressure gradient $\nabla P$ cannot exceed $4 \sigma / e L^{\prime}$, with $L^{\prime}$ the typical length between two rows of bubbles (Fig. 5): $L^{\prime}=3 b / 2$ with $b$ the side of the hexagons. The bubble area is then $\bar{S}=\pi L^{2}=3 b^{2} \sqrt{3} / 2$, thus $L^{\prime} / L=3^{1 / 4} \sqrt{\pi / 2} \simeq 1.65$. Now, force balance imposes $\nabla P=2 \tau_{w} / e$, hence the maximal velocity obeys:

$$
\frac{2 \sigma}{L^{\prime}}=\tau_{w},
$$

where $\tau_{w}$ is given by (3). This gives an implicit prediction of the maximal velocity as a function of the liquid fraction, that we plot in Fig. 4b. Although the qualitative variation with the liquid fraction is right, this prediction largely overestimates (by a factor 4) the experimental data.

We do not have a quantitative reason for such a large discrepancy, but we can at least provide possible reasons. Actually, just before bursting, the bubbles display huge deviations from their quasistatic shape (Fig. 3). We have observed that the bubbles that are closest to a growing tip of the opening bubble, which are the ones that move the fastest, are extremely 


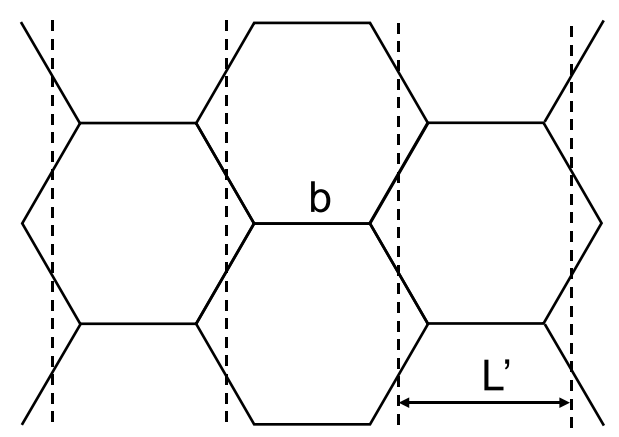

Figure 5: Sketch of a hexagonal foam ( $b$ : side of the hexagons), showing the definition of the distance $L^{\prime}$ between two rows of bubbles.

elongated in the transverse direction (Fig. 3c). For such bubbles, the Plateau rules of equilibrium at clearly violated: the curvature along a given edge is not constant and can even revert, and three bubble edges do not meet at $120^{\circ}$. Interestingly, all these observations corroborate recent numerical studies $[22,23,24,25]$, that evidenced that non-quasistatic effects delay the occurrence of bubble rearrangements and enhance bubble deformation. More precisely, the way bubble edges meet suggests that edges perpendicular to the tip motion have a much larger tension than the short edges parallel to it (Fig. 3c). All together, this shows that the assumption of a constant surface tension clearly breaks down when bubbles get so distorted that they are about to burst. It invalidates expression (3), which assumes small relative surface tension variations: $|\delta \sigma| / \sigma \ll 1[20]$. Still, the fact that (3) predicts the opening speed (Fig. 2) probably means that the overall friction is dominated by zones where $|\delta \sigma| / \sigma \ll 1$, which is consistent with the fact that most of the foam is not much distorted in Fig. 3a,b.

\subsection{Minimal velocity in the fragile regime}

Consistently with [1], we have also found that the fragile cracks do not propagate slower than a certain velocity. To measure such a minimal velocity, we have considered experiments where a spontaneous fragile-ductile transition occurs, as considered also in [15]; we then take the displacement of the crack tip between the two images in the fragile regime closest to the transition as an estimate of the minimal velocity of fragile propagation $v_{\min }$. There is a significant uncertainty in this measurement, since the displacement of the fragile crack tip is quantised by the number of broken films between two consecutive images, which is usually 3 or 4 ; hence, we ascribe a rela- 

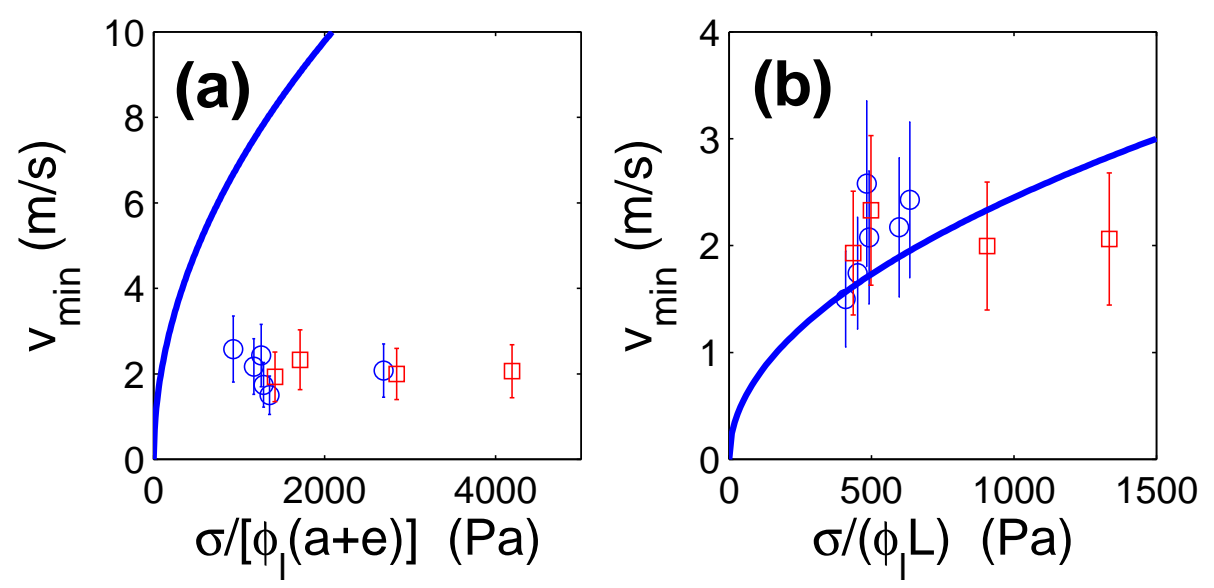

Figure 6: Experimental minimal velocity of propagation of the brittle cracks, for the SDS solutions (o) and the SLES/CAPB/MAc mixture ( $\square$ ) as a function of: (a) the parameter $\sigma /\left[\phi_{\ell}(a+e)\right]$, (b) the parameter $\sigma / \phi_{\ell} L$, both expressed in Pa. The curves are: (a) the prediction (8), (b) the propagation speed of shear waves in a 2D foam, Eq. (7).

tive uncertainty of $30 \%$ on $v_{\min }$. These velocities are plotted in Fig. 6, for experiments with different liquid fractions and surfactant solutions.

We now turn to the comparison with the speed of shear waves, which equals [26]:

$$
c_{S}=\sqrt{\frac{6 \sigma}{\rho \phi_{\ell} L}} .
$$

This expression assumes that all the liquid contained within the foam is entrained into motion. On the contrary, Hilgenfeldt and colleagues [1] argued that only the vertical Plateau borders, orthogonal to the plates, move, whereas the horizontal Plateau borders in contact with a plate are stuck because of the no-slip boundary condition at the plates. In this case, Eq. (7) is replaced by:

$$
c_{S}=\sqrt{\frac{6 \sigma}{\rho \phi_{\ell}^{\prime} L}} .
$$

with an effective liquid fraction, accounting for the vertical Plateau borders only: $\phi_{\ell}^{\prime} \simeq(e+a) A_{\text {vert }} \phi_{\ell} / 3 L A_{\text {hor }}$, where $A_{\text {vert }}=(\sqrt{3}-\pi / 2) a^{2}$ and $A_{\text {hor }}=(2-$ $\pi / 2) a^{2}$ are the cross-sections of vertical and horizontal Plateau borders; see [1] for a derivation of $\phi_{\ell}^{\prime}$. There is a significant uncertainty in this definition of $\phi_{\ell}^{\prime}$, since the length of the vertical Plateau borders is of order $e$ but not 
well defined, and also because we use $L$ and not $b$ as a characteristic bubble size, leading to a relative uncertainty $b / L-1=3^{-3 / 4} \sqrt{2 \pi}-1=10 \%$.

Contrary to the maximal velocity of ductile crack propagation, these criteria do not depend on interfacial rheology, hence we can test data on both solutions simultaneously. We report prediction (8) on Fig. 6a, and prediction (7) on Fig. 6b, together with the data. It shows that (8) overestimates the minimal velocities observed, whereas (7) is a very efficient lower bound, except for two data points. Hence, if our measurements tend to confirm the idea of the speed of shear waves as a minimal velocity of fragile crack propagation, they are compatible with the fact that all the liquid within the foam is put into motion. Actually, in our experiments, the time $t$ at which the minimal velocity is measured after the air injection starts is very short, always less than $10^{-2} \mathrm{~s}$. But over such a time, the liquid is entrained at a constant speed across the gap, except for a thin boundary layer of thickness $\delta \approx \sqrt{\eta t / \rho}$ close to the plates, which is the thickness over which the no-slip boundary condition is "felt" by the liquid. In our experiments, since $t<10^{-2} \mathrm{~s}, \delta$ is less than $0.1 \mathrm{~mm}$, whereas the Plateau border radius is $a \simeq 0.2 \mathrm{~mm}$ in the experiments on Fig. 6. Hence, a large part of the liquid in the horizontal Plateau border is still not affected by the boundary condition when the minimal velocity is measured, which is probably why (7) fits better our data than (8).

\section{Description of branched fracture}

Once we inject air far above the onset of fragile fracture, we have observed that branching occurs: secondary cracks detach and grow from already created cracks. This phenomenon is ubiquitous in fracture of brittle materials $[27,28]$. We do not intend to give any prediction on the onset of branching; it is worth noting, though, that criteria based on a critical velocity lower than the speed of shear waves $[29,28]$ are likely to fail in the case of brittle fracture of foams, if the latter is indeed "supersonic" (Sec. 4.2).

A general observation is that the degree of branching increases with increasing overpressure. We illustrate this behaviour in Fig. 7, where the crack centrelines are shown for four different experiments. We have observed that brittle cracks propagate as a narrow line of only one bubble in thickness; hence, branching occurs as soon as a single bubble bursts aside the main crack, which corresponds to the shortest branches in Fig. 7.

We have also measured in this branched regime $v_{\min }$ as described in Sec. 4.2. We have found neither a significant dependence on the applied 


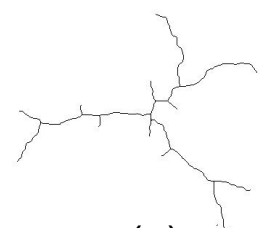

(a)

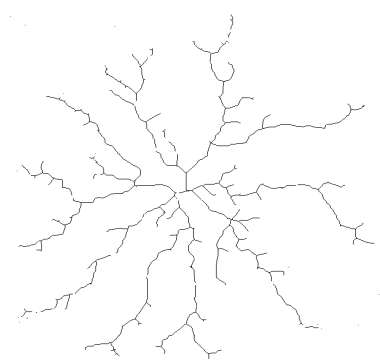

(c)

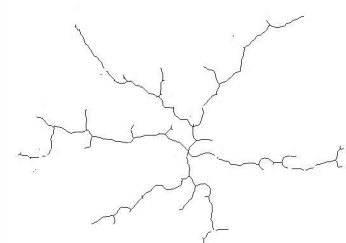

(b)

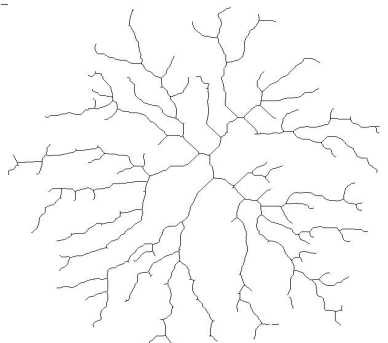

(d)

Figure 7: Crack centrelines for four experiments in the branched fragile regime, at increasing overpressure: (a) $3.3 \mathrm{kPa}$, (b) $6.0 \mathrm{kPa}$, (c) $60 \mathrm{kPa}$ and (d) $120 \mathrm{kPa}$.

overpressure (between 3.3 and $120 \mathrm{kPa}$ ), nor a significant difference between different branches on a given experiment.

\section{Conclusions}

We have given complementary results on the response of a $2 \mathrm{D}$ foam to air injection. Together with previous studies [1, 17], they provide a good understanding of several aspects, like the existence of critical velocities for the ductile and the fragile regimes, and the injection rate in the ductile regime. However, they raise new interesting questions. Specifically, the study of the maximal velocity withstood by a foam with incompressible interfaces shows the need for a further study of its rheology far from equilibrium, which requires to consider explicitly surface tension variations for a more complete modelling of boundary conditions. In the fragile case, it is not fully clear why $c_{S}$ is involved in the criterion instead of $c_{L}$, which would tend to equilibrate the pressures much faster than the observed crack propagation. Finally, branching should be modelled in a context of "supersonic" fracture, which is quite unusual for most brittle materials. 
In a different direction, it would be interesting to extend this study to the 3D case. This poses a major technical challenge, given the opacity of 3D foams, requiring the use of advanced imaging techniques such as X-ray tomography. However, this could bring some insight on the remarkable ability of 3D foams to absorb the energy released by the sudden injection of gas or pressure, as in shock waves or explosions.

\section{References}

[1] S. Arif, J. C. Tsai, S. Hilgenfeldt, Speed of crack propagation in dry aqueous foams, EPL 92, 38001 (2010).

[2] I. Cantat, S. Cohen-Addad, F. Elias, F. Graner, R. Höhler, O. Pitois, F. Rouyer, A. Saint-Jalmes, Les mousses. Structure et dynamique, Belin, Paris (2010).

[3] N. D. Denkov, S. Tcholakova, K. Golemanov, K. P. Ananthapadmanabhan, A. Lips, The role of surfactant type and bubble surface mobility in foam rheology, Soft Matter 5, 3389-3408 (2009).

[4] G. Katgert, B. P. Tighe, M. E. Möbius, M. van Hecke, Couette flow of two-dimensional foams, EPL 90, 54002 (2010).

[5] I. Cheddadi, P. Saramito, B. Dollet, C. Raufaste, F. Graner, Understanding and predicting viscous, elastic, plastic flows, Eur. Phys. J. E 34, 1 (2011).

[6] P. Schall, M. van Hecke, Shear bands in matter with granularity, Ann. Rev. Fluid Mech. 42, 67-88 (2010).

[7] L. Bocquet, A. Colin, A. Ajdari, Kinetic theory of plastic flow in soft glassy materials, Phys. Rev. Lett. 103, 036001 (2009).

[8] R. Raspet, S. K. Griffiths, The reduction of blast noise with aqueous foams, J. Acoust. Soc. Am. 74, 1757-1763 (1983).

[9] A. Britan, M. Liverts, G. Ben-Dor, Mitigation of sound waves by wet aqueous foams, Colloids Surf. A 344, 48-55 (2009).

[10] E. Del Prete, A. Chinnayya, L. Domergue, A. Hadjadj, J. F. Haas, Blast wave mitigation by dry aqueous foams, to appear in Shock Waves, DOI 10.1007/s00193-012-0400-0. 
[11] B. Dollet, I. Cantat, Deformation of soap films pushed through tubes at high velocity, J. Fluid Mech. 652, 529-539 (2010).

[12] I. Cantat, B. Dollet, Liquid films with high surface modulus moving in tubes: dynamic wetting film and jumpy motion, Soft Matter 8, 77907796 (2012).

[13] S. S. Park, D. J. Durian, Viscous and elastic fingering instabilities in foam, Phys. Rev. Lett. 72, 3347-3350 (1994).

[14] A. Lindner, P. Coussot, D. Bonn, Viscous fingering in a yield stress fluid, Phys. Rev. Lett. 85, 314-317 (2000).

[15] S. Arif, J. C. Tsai, S. Hilgenfeldt, Spontaneous brittle-to-ductile transition in aqueous foam, J. Rheol. 56, 485-499 (2012).

[16] E. Bouchbinder, J. Fineberg, M. Marder, Dynamics of simple cracks, Ann. Rev. Condens. Matter Phys. 1, 371-395 (2010).

[17] I. Ben Salem, I. Cantat, B. Dollet, Response of a two-dimensional liquid foam to air injection: swelling rate, fingering and fracture, J. Fluid Mech. 714, 258-282 (2013).

[18] K. Golemanov, N. D. Denkov, S. Tcholakova, M. Vethamuthu, A. Lips, Surfactant mixtures for control of bubble surface mobility in foam studies, Langmuir 24, 9956-9961 (2008).

[19] N. D. Denkov, S. Tcholakova, K. Golemanov, V. Subramanian, A. Lips, Foam-wall friction: Effect of air volume fraction for tangentially immobile bubble surface, Colloids Surf. A 282, 329-347 (2006).

[20] I. Cantat, Liquid meniscus friction on a wet plate: Bubble, lamella and foam, submitted.

[21] P. Grassia, G. Montes-Atenas, L. Lue, T. E. Green, A foam film propagating in a confined geometry: Analysis via the viscous froth model, Eur. Phys. J. E 25, 39-49 (2008).

[22] T.E. Green, P. Grassia, L. Lue, B. Embley, Viscous froth model for a bubble staircase structure under rapid applied shear: An analysis of fast flowing foam, Colloids Surf. A 348, 49-58 (2009). 
[23] I. Cantat, Gibbs elasticity effect in foam shear flows: a non quasi-static 2D numerical simulation, Soft Matter 7, 448-455 (2011).

[24] B. Embley, P. Grassia, Viscous froth simulations with surfactant mass transfer and Marangoni effects: Deviations from Plateau's rules, Colloids Surf. A 382, 8-17 (2011).

[25] P. Grassia, B. Embley, C. Oguey, A Princen hexagonal foam out of physicochemical equilibrium, J. Rheol. 56, 501-526 (2012).

[26] L. W. Schwartz, H. M. Princen, A theory of extensional viscosity for flowing foams and concentrated emulsions, J. Colloid Interface Sci. 118, 201-211 (1987).

[27] E. Sharon, S. P. Gross, J. Fineberg, Local crack branching as a mechanism for instability in dynamic fracture, Phys. Rev. Lett. 74, 5096-5099 (1995).

[28] E. Bouchbinder, J. Mathiesen, I. Procaccia, Branching instabilities in rapid fracture: Dynamics and geometry, Phys. Rev. E 71, 056118 (2005).

[29] M. Adda-Bedia, Brittle fracture dynamics with arbitrary paths III. The branching instability under general loading, J. Mech. Phys. Solids 53, 227-248 (2005). 